\title{
BMJ Open Guidelines for the symptomatic management of fever in children: systematic review of the literature and quality appraisal with AGREE II
}

\author{
Elena Chiappini, Barbara Bortone, Luisa Galli, Maurizio de Martino
}

To cite: Chiappini E, Bortone B, Galli L, et al. Guidelines for the symptomatic management of fever in children: systematic review of the literature and quality appraisal with AGREE II. BMJ Open 2017;7:e015404. doi:10.1136/ bmjopen-2016-015404

- Prepublication history and additional material are available. To view these files please visit the journal online (http://dx.doi. org/10.1136/bmjopen-2016015404).

Received 3 December 2016

Revised 22 May 2017

Accepted 8 June 2017

\section{CrossMark}

Department of Health Sciences, University of Florence, Anna Meyer Children's University Hospital, Florence, Italy

Correspondence to Dr Elena Chiappini; elena.chiappini@unifi.it

\section{ABSTRACT}

Introduction Several societies have produced and disseminated clinical practice guidelines (CPGs) for the symptomatic management of fever in children. However, to date, the quality of such guidelines has not been appraised.

Objective To identify and evaluate guidelines for the symptomatic management of fever in children. Methods The research was conducted using PubMed, guideline websites, and Google (January 2010 to July 2016). The quality of the CPGs was independently assessed by two assessors using the Appraisal of Guidelines for Research \& Evaluation II (AGREE II) instrument, and specific recommendations in guidelines were summarised and evaluated. Domain scores were considered of sufficient quality when $>60 \%$ and of good quality when $>80 \%$.

Results Seven guidelines were retrieved. The median score for the scope and purpose domain was $85.3 \%$ (range 66.6-100\%). The median score for the stakeholder involvement domain was 57.5\% (range 33.3-83.3\%) and four guidelines scored $>60 \%$. The median score for the rigour of development domain was $52.0 \%$ (range 14.6-98.9\%), and only three guidelines scored $>60 \%$. The median score for the clarity of presentation domain was $80.9 \%$ (range $50.0-94.4 \%$ ). The median score for the applicability domain was $39.3 \%(8.3-100 \%)$. Only one guideline scored $>60 \%$. The median score for the editorial independence domain was $48.84 \%$ (0-91.6\%); only three guidelines scored $>60 \%$.

Conclusion Most guidelines were recommended for use even if with modification, especially in the methodology, the applicability and the editorial independence domains. Our results could help improve reporting of future guidelines, and affect the selection and use of guidelines in clinical practice.

\section{INTRODUCTION}

Fever is one of the most common clinical reasons for paediatric consultations, accounting for about one-third of all presenting conditions in children. ${ }^{1-3}$

Concerns of parents/tutors/caregivers about serious causes of fever (ie, severe bacterial infections) and misconceptions
Strengths and limitation of this study

- To the best of our knowledge this is the first study to appraise guidelines on the symptomatic management of fever with the AGREE II instrument.

- Moreover, recommendations dealing with symptomatic management of fever have been extracted and resumed in comparative tables, focusing on possible gaps and common messages between the guidelines.

- The AGREE methodology does not provide a threshold for discrimination between high quality and low quality guidelines.

- Searching may not have been exhaustive; therefore, we cannot exclude the possibility that some guidelines may have been omitted from this study.

about fever as a sufficient trigger of brain damage have led to the spreading of 'feverphobia'. ${ }^{45}$ Several studies have reported a high percentage of parents/tutors/caregivers administering antipyretics even when there is minimal or no fever, with wrong dosages or with insufficient intervals between the doses. ${ }^{4-6}$ Fever is a physiologic mechanism with beneficial effects in fighting infection and it is not associated with long-term neurologic complications. ${ }^{7}$ The only purpose for treating fever in children must be to relieve the child's discomfort and not to lower the body temperature. ${ }^{8}$

The inappropriate management of fever may delay the diagnosis and increase the risk of antipyretic overdose. Moreover, other factors may increase drug toxicity such as the alternate/combined use of two antipyretics, ${ }^{9}$ the use of rectal formulations, ${ }^{10}$ and the administration of these drugs in the presence of contraindicated underlying diseases. ${ }^{11}$ Finally, overtreatment may have a significant economic impact in low-middle income and high income countries. 
In order to rationalise and standardise the symptomatic management of fever in children, national health agencies and scientific societies have produced and disseminated clinical guidelines. It has been demonstrated that parents/tutors do not fully comply with these recommendations, as they used to employ traditional physical means and administer antipyretics with inappropriate indications and posology. ${ }^{32-16}$ Moreover, important discrepancies have been reported between the practices of healthcare professionals and the recommendations of guidelines. ${ }^{17-19}$

Several barriers to applying these guidelines in clinical practice have been identified. ${ }^{19-22}$ Thus, we conducted this study to identify and evaluate the quality of the international clinical practice guidelines (CPGs) for the use of antipyretics and physical methods in children with fever, focusing on discrepancies.

\section{METHODS}

\section{Primary outcomes}

The primary outcomes of the study were to encourage the improvement of the quality of guidelines, to reinforce the messages of common recommendations, and to stimulate further research on discordant recommendations and the issues of international guidelines in order to unify medical behaviour.

\section{Guidelines research}

The search for guidelines for the symptomatic management of fever in children was carried out using documents issued by national scientific societies or by government organisations between January 2010 and July 2016 in every language, through the use of appropriate keywords and the following search engines: PubMed; National Guideline Clearinghouse (www.guideline.gov); NICE: National Institute for Health and Care Excellence (www.nice.org.uk); Canadian CPG Infobase: Clinical Practice Guidelines Database (www.cma.ca/En/Pages/ clinical-practice-guidelines.aspx); Scottish Intercollegiate Guidelines Network (SIGN) (www.sign.ac.uk); Australian Clinical Practice Guidelines (http://www.clinicalguidelines.gov.au/);\%20and Guidelines International Network (http://www.g-i-n.net/). Additional research was conducted on Google. In this research we used the following keywords: 'guideline', 'fever', 'children', and 'antipyretics', (see 'Search strategy' in supplementary material 1). The search was limited to January 2010 and not earlier in order to evaluate only the most recent and updated guidelines.

\section{Exclusion criteria}

Guidelines that did not focus on the management of fever as a symptom/sign, or were not original or were issued on a regional level, were excluded, as well as any documents that were not guidelines (such as position papers and reviews).

\section{Quality evaluation}

Two assessors, one with experience in developing and evaluating guidelines (EC) and another assessor (BB), used the online training tools recommended by the AGREE collaboration before conducting appraisals. ${ }^{23}$ They independently evaluated the included guidelines using the AGREE II instrument,${ }^{23}$ which consists of a total of 23 items in six domains: 'Scope and purpose', 'Stakeholder involvement', 'Rigour of development', 'Clarity of presentation', 'Applicability', and 'Editorial independence'. Each item was rated on a seven-point scale from 1 (strongly disagree) to 7 (strongly agree). A scaled domain percentage score was calculated, according to the AGREE II methodology, ${ }^{23}$ as follows: (obtained score-minimum possible score)/(maximum possible score-minimum possible), where the 'obtained score' is the sum of the appraisers scores per each item, making it possible to consider the natural discrepancies between the two appraisers.

Although the domain scores are useful for comparing guidelines and attest whether a guideline should be recommended for use, the AGREE II instrument does not set minimum domain scores or patterns of scores across domains to differentiate between high quality and poor quality guidelines. These decisions should be made by the users and guided by the context in which AGREE II is being used. ${ }^{23}$ Then, as reported in previous studies, ${ }^{24}{ }^{25}$ we considered a value $>60 \%$ as sufficient quality score and a value $>80 \%$ as a good quality score.

On completing the 23 items, the appraisers provided the overall assessment of each guideline, and decided which guideline was recommendable, with or without modifications, and which was not recommendable. This choice was the result of the six domains' scores and of the personal judgement of the appraisers. To resolve discrepancies between the two assessors, a method was used from a previous study: if the scores assigned by the assessors differed by 1 point the lower score was used; if they differed by 2 points they were averaged; and if they varied by $\geq 3$ points a consensus was reached after a discussion. ${ }^{24}$ We decided to recommend without modification the guidelines with an overall score equal to 7, to recommend with modification the guidelines with an overall score $\geq 4$ but $<7$, and not to recommend the guidelines with an overall score $\leq 3$.

\section{Comparison of recommendations}

Recommendations regarding the symptomatic management of fever in children, reported in the selected guidelines, have been extracted and summarised in comparative tables focusing on possible gaps and common messages.

\section{RESULTS}

\section{Guideline selection}

A total of 122 records were initially identified, of which, after screening titles and abstracts, 97 were excluded because they were irrelevant or duplicates. The remaining 25 records were retrieved for full text. ${ }^{26-49}$ Among these, 18 documents were excluded because they were 


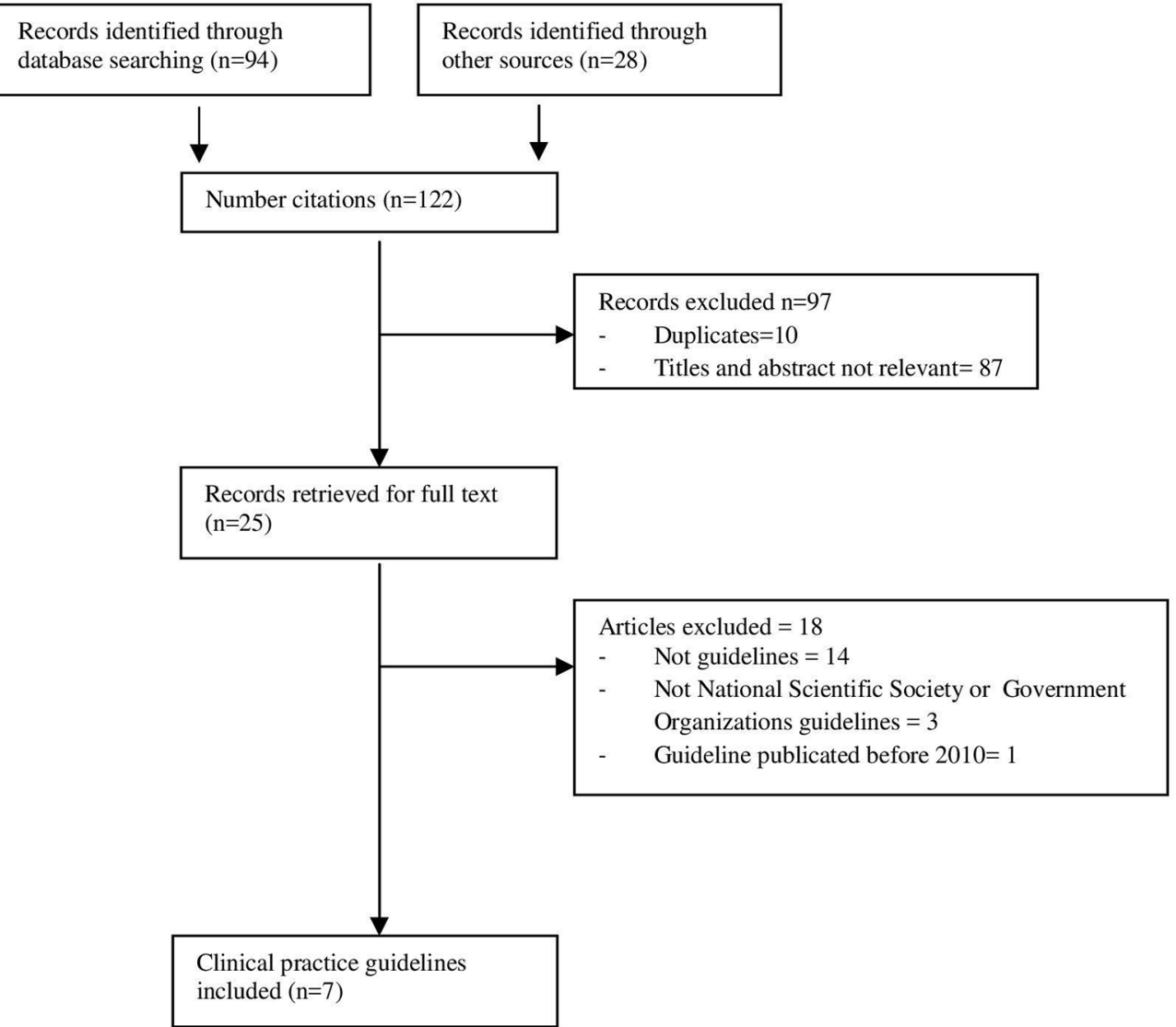

Figure 1 Flow chart of searching and selecting guidelines.

not guidelines 262829 31-38 40-42 or they were not medical societies' or health government guidelines ${ }^{394346}$; one of the guidelines was excluded because the original publication date was before 2010. ${ }^{45}$ Finally, seven CPGs were selected $^{2} 27304447-49$ (figure 1). The characteristics of the included guidelines are presented in table 1 .

\section{AGREE II scores}

The domain-standardised scores for selected guidelines and overall recommendations are presented in table 2 and figure 2.

The American Academy of Pediatrics (AAP) ${ }^{2}$ guideline has good scores in clarity of presentation and editorial independence domains, sufficient score in scope and purpose, with low scores in stakeholder involvement, rigour of development and applicability domains.

The Italian Pediatric Society $(\mathrm{SIP})^{27}$ guideline has good scores in scope and purpose, stakeholder involvement, rigour of development, clarity of presentation and editorial independence domains with a low score in applicability domain.

The South African ${ }^{30}$ guideline has good scores in scope and purpose and clarity of presentation, sufficient score in stakeholder involvement, but low scores in rigour of development, applicability and editorial independence domains.

The National Institute for Health and Care Excellence (NICE) ${ }^{44}$ guideline has good scores in scope and purpose, stakeholder involvement, rigour of development, clarity of presentation and applicability domains with a low score in editorial independence.

The New South Wales Ministry of Health (NSW) ${ }^{47}$ guideline has a sufficient score in the scope and purpose domain, but low scores in all the others.

The South Australian Ministry of Health (SA) ${ }^{48}$ guideline has a good score in scope and purpose, a sufficient score in clarity of presentation domain, but low scores in the others.

The $\mathrm{WHO}^{49}$ guideline has good scores in scope and purpose, rigour of development, clarity of presentation and sufficient scores in stakeholder involvement, applicability and editorial independence domains.

\section{Scope and purpose}

The median score for the scope and purpose domain was $85.28 \%$ (range $66.6-100 \%$ ).

Most guidelines clearly described their overall objectives, health questions and target populations. The NSW guideline has the lowest score. ${ }^{47}$

\section{Stakeholder involvement}

The median score for the stakeholder involvement domain was $57.5 \%$ (range 33.30-83.30\%). Only the SIP, South African, NICE, and WHO guidelines scored above $60 \%$ for this domain, ${ }^{27} 304449$ whereas the AAP, NSW and SA guidelines did not consider the views and preferences of the target population. ${ }^{2478}$ No guideline clearly described their members' roles in the guideline 


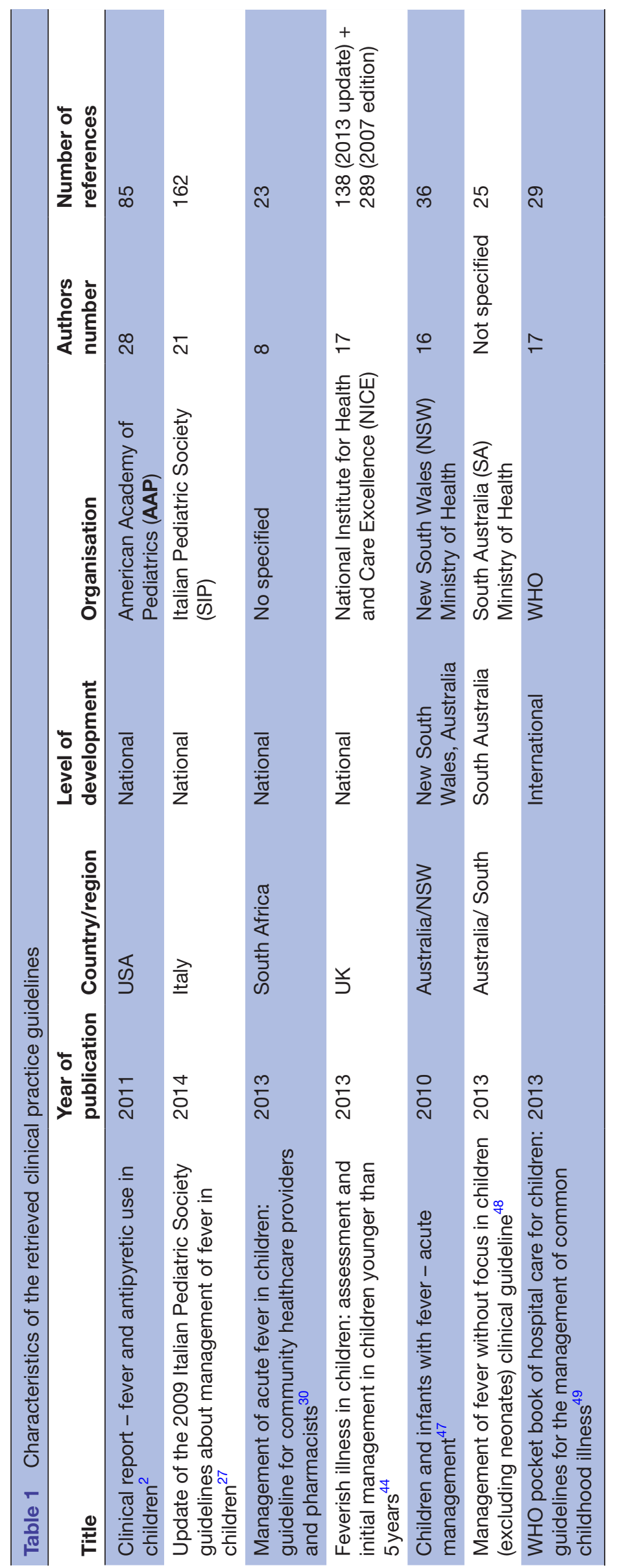

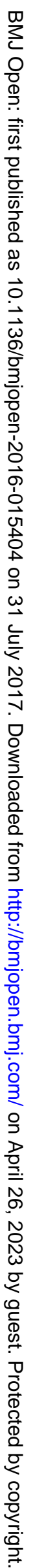


Table 2 Standardised scores of each domain by AGREE II of guidelines

\begin{tabular}{|c|c|c|c|c|c|c|c|}
\hline & $\begin{array}{l}\text { Scope and } \\
\text { purpose }\end{array}$ & $\begin{array}{l}\text { Stakeholder } \\
\text { involvement }\end{array}$ & $\begin{array}{l}\text { Rigour of } \\
\text { development }\end{array}$ & $\begin{array}{l}\text { Clarity of } \\
\text { presentation }\end{array}$ & Applicability & $\begin{array}{l}\text { Editorial } \\
\text { Independence }\end{array}$ & Overall assessment \\
\hline $\mathrm{AAP}^{2}$ & $75 \%$ & $41.60 \%$ & $23.90 \%$ & $83.30 \%$ & $27.10 \%$ & $83.30 \%$ & $\begin{array}{l}\text { 5. Recommended with } \\
\text { modifications }\end{array}$ \\
\hline $\operatorname{SIP}^{27}$ & $94.40 \%$ & $80.50 \%$ & $89.60 \%$ & $94.40 \%$ & $27.10 \%$ & $91.60 \%$ & $\begin{array}{l}\text { 6. Recommended with } \\
\text { modifications }\end{array}$ \\
\hline $\begin{array}{l}\text { South } \\
\text { African }^{30}\end{array}$ & $88.80 \%$ & $61.10 \%$ & $31.20 \%$ & $83.30 \%$ & $8.30 \%$ & $50 \%$ & $\begin{array}{l}\text { 4. Recommended with } \\
\text { modifications }\end{array}$ \\
\hline $\mathrm{NICE}^{44}$ & $100 \%$ & $83.30 \%$ & $98.90 \%$ & $83.30 \%$ & $100 \%$ & $50 \%$ & 7. Recommended \\
\hline $\mathrm{NSW}^{47}$ & $66.60 \%$ & $41.60 \%$ & $16.00 \%$ & $50 \%$ & $33.30 \%$ & $0 \%$ & 3. Not recommended \\
\hline $\mathrm{SA}^{48}$ & $83.30 \%$ & $33.30 \%$ & $14.60 \%$ & $77.70 \%$ & $16.60 \%$ & $0 \%$ & 3. Not recommended \\
\hline $\mathrm{WHO}^{49}$ & $88.90 \%$ & $61.10 \%$ & $89.60 \%$ & $94.40 \%$ & $62.50 \%$ & $67 \%$ & $\begin{array}{l}\text { 6. Recommended with } \\
\text { modifications }\end{array}$ \\
\hline
\end{tabular}

AAP, American Academy of Pediatrics; NICE, National Institute for Health and Care Excellence; NSW, New South Wales Ministry of Health; SA, South Australian Ministry of Health; SIP, Italian Pediatric Society.

development process. Only the SIP and NICE guidelines had methodology experts included in the guideline development group. ${ }^{2744}$ Only in the NICE guideline were there health economists among the guideline authors. ${ }^{44}$

\section{Rigour of development}

The median score for the rigour of development domain was $51.97 \%$ (range $14.60-98.90 \%$ ). Only the SIP, NICE, and WHO guidelines scored $>60 \%$ because they used systematic methods of searching for evidence and for formulating recommendations ${ }^{27} 4449$; only the
NICE and WHO CPGs clearly described methods for conducting external reviews ${ }^{44}{ }^{49}$; and only the NICE and WHO CPGs described their procedures for updating guidelines. ${ }^{44} 49$

\section{Clarity of presentation}

Most guidelines provided specific, unambiguous and easily identifiable recommendations. The median score for the clarity of presentation domain was $80.9 \%$ (range 50-94.4\%). Only the NSW guideline scored $<60 \%{ }^{47}$

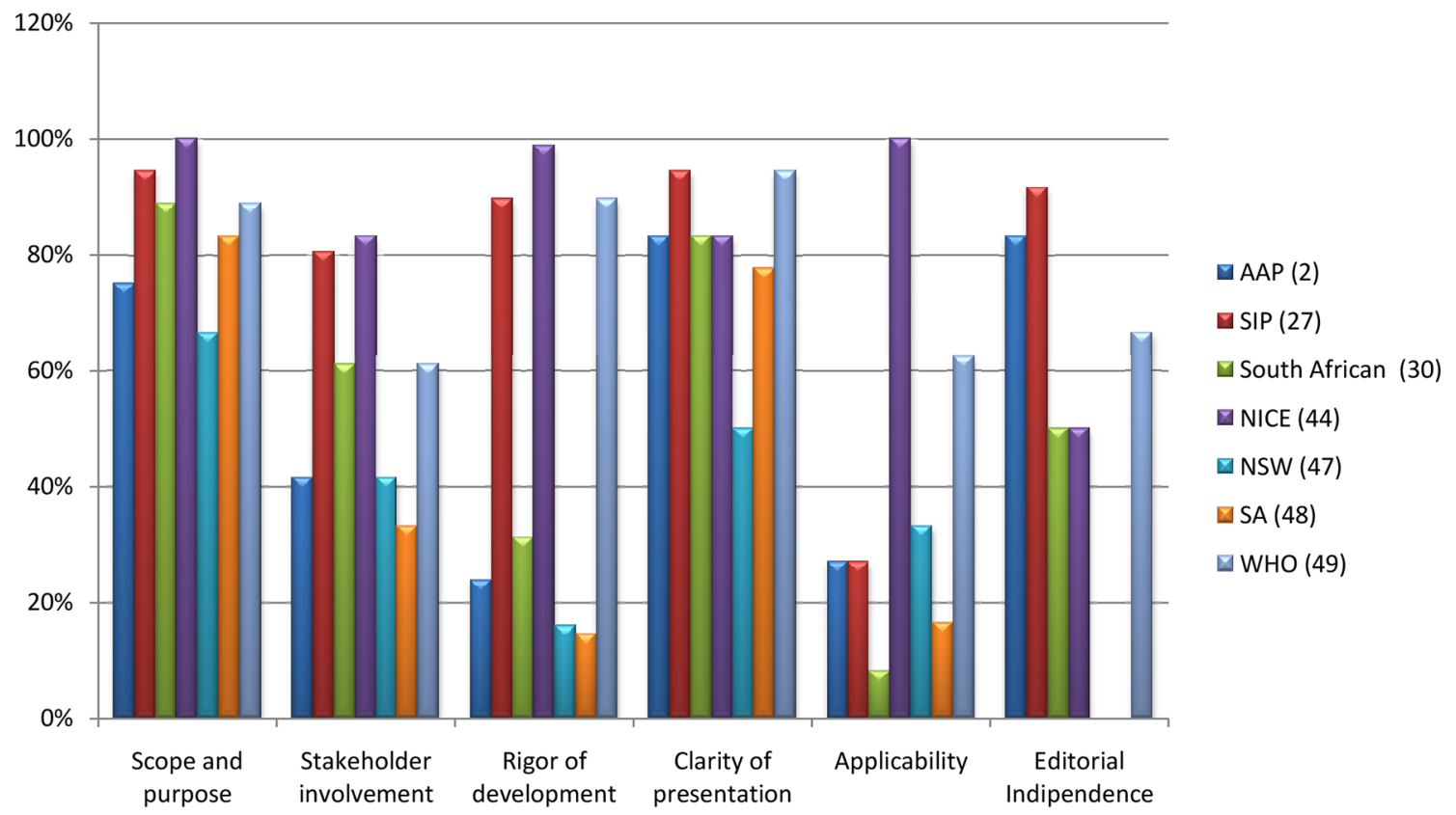

AAP: American academy of pediatrics; SIP: Italian Pediatric Society; NICE: National institute for Health and clinical excellence; NSW: New South Wales ministry of Health; SA: South Australian Ministry of Health; WHO: World Health Organization

Figure 2 Standardised scores of each domain by AGREE II of guidelines-histogram. 


\section{Applicability}

The median score for the applicability domain was $39.3 \%$ $(8.3-100 \%)$. Only the NICE guideline scored $>60 \%{ }^{44}$ Most guidelines did not describe the facilitators and barriers of their applications and did not sufficiently consider the costs of applying their recommendations; only the NICE guideline involved a health economist in finding and analysing cost information. ${ }^{44}$ Only the AAP and SA guidelines did not provide any tool or suggestions for putting the recommendations into practice. ${ }^{248}$

\section{Editorial independence}

The median score for the editorial independence domain was $48.84 \%$ (0-91.6\%); only the AAP, SIP and WHO CPGs scored $>60 \% .{ }^{227} 49$ The NWS and SA CPGs did not clearly provide financial support information. ${ }^{47}$ Although the WHO guideline reported the funding source, it did not specify the possible funding influence on the CPG content. ${ }^{49}$ Moreover, only the AAP, SIP and WHO guidelines include a conflict of interest statement by the guideline development group. ${ }^{27} 49$

\section{Overall assessment}

Based on the six domain scores and on a personal judgement, the AAP, SIP, South African and WHO guidelines were recommended with modifications, ${ }^{2} 273049$ only the NICE CPG was recommended without modifications, ${ }^{44}$ and the NSW and SA CPGs were not recommended. ${ }^{47} 48$

\section{Summary of recommendations}

Specific recommendations have been identified and summarised in table 3 , as well as common and discordant messages.

\section{Common messages}

1. Antipyretics are indicated only in cases of discomfort associated with fever and not with the sole aim of reducing body temperature.

2. Recommended antipyretics are paracetamol or ibuprofen, according to the child's age, weight and characteristics.

3. The use of antipyretics does not prevent either febrile convulsions or reactions to vaccines.

4. Tepid sponging and alcoholic baths are not recommended for the treatment of fever.

5. The use of cough and cold medicine is discouraged because of the risks of overdoses and interactions.

6. Caution is recommended using antipyretics in chronic diseases such as pre-existing hepatic and renal impairment or in cases of diabetes, cardiac disease and severe malnutrition.

7. In asthmatic children with fever, paracetamol does not seem to worsen asthma symptoms.

\section{Divergent messages}

1. The physical method of unwrapping/uncovering children with fever is contraindicated by the SIP, South African and NICE CPGs ${ }^{25} 28$ and indicated by the NWS and WHO CPGs. ${ }^{47} 49$
2. The alternate use of two antipyretics is discouraged by most of the guidelines with the exception of the NICE and SA CPGs. ${ }^{448}$ These two guidelines permit the alternate use only if the discomfort persists after the administration of one antipyretic. ${ }^{44} 48$

3. The minimum age for administering paracetamol varies from birth (SIP, NWS, $\mathrm{SA}^{274748}$ ) to 2 months $\left(\mathrm{WHO}^{49}\right.$ ), to 3 months (South African ${ }^{30}$ ) (online supplementary file 2).

4. The posology of paracetamol varies in terms of dosage per single administration (10-15 or $15 \mathrm{mg}$ / $\mathrm{kg} /$ dose), of intervals between doses (4hour, 4-6 hour or 6 hour) and of maximum allowable daily dosage that ranges from $60 \mathrm{mg} / \mathrm{kg} /$ day to $90 \mathrm{mg} / \mathrm{kg}$ / day (online supplementary file 2 ).

5. The posology of paracetamol in newborns ${ }^{27} 48$ varies by paracetamol dosage per single administration and maximum daily dosages in newborns (online supplementary file 3 ).

6. The minimum age for ibuprofen administration ranges from 2 to 3 months (NWS, ${ }^{47} \mathrm{WHO}^{49}$ ) to 6 months $\left(\mathrm{AAP}^{2}\right)$ (online supplementary file 4 ).

7. The posology of ibuprofen is divergent in terms of dosage per single administration $(5-10$ or $10 \mathrm{mg} / \mathrm{kg}$ / dose), of intervals between doses (6, 6-8hours) and maximum daily dosage of $40 \mathrm{mg}$ for all guidelines except the SIP CPG that allows up to $30 \mathrm{mg}^{27}$ (online supplementary file 4 ).

8. The use of ibuprofen in asthmatic patients is contraindicated in the SIP CPG only in patients with asthma related to non-steroidal anti-inflammatory drugs but indicated with caution in all asthmatic patients by the South African and SA CPGs. ${ }^{30} 48$

9. The use of ibuprofen in children with dehydration is contraindicated by the SIP CPG,${ }^{27}$ but recommended with caution by the AAP, South African and SA CPGs. ${ }^{2} 30$

10. The use of ibuprofen in the case of varicella is contraindicated by the SIP $\mathrm{CPG}^{27}$ and advised with caution by the AAP and South African CPGs. ${ }^{230}$

\section{DISCUSSION AND CONCLUSIONS}

In the present study a systematic search of the available national guidelines regarding the management of fever in children was performed, and key messages have been summarised. Overall, only seven guidelines were retrieved, which is less than expected considering that fever represents a large part of the practice of healthcare professionals, and how common the use of these drugs is. In particular, only two guidelines (AAP, SIP $^{27}$ ) focused specifically on the symptomatic management of feverish children, whereas the other five guidelines dealt with the other aspects of fever management, including aetiological diagnosis and therapy.

Considering the impact of the quality of the guidelines in their application, we appraised the quality scores of the CPGs with the AGREE II instrument. 


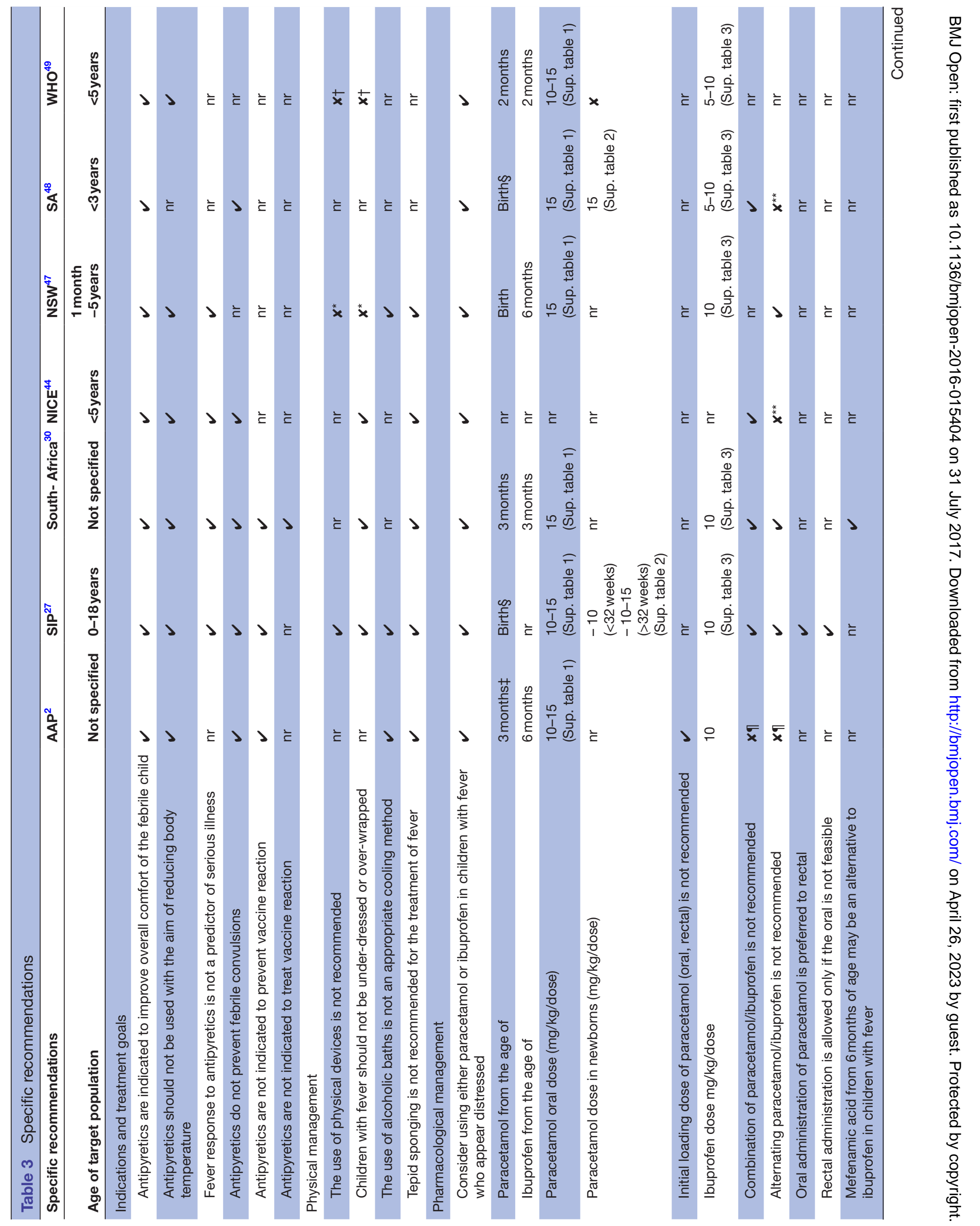




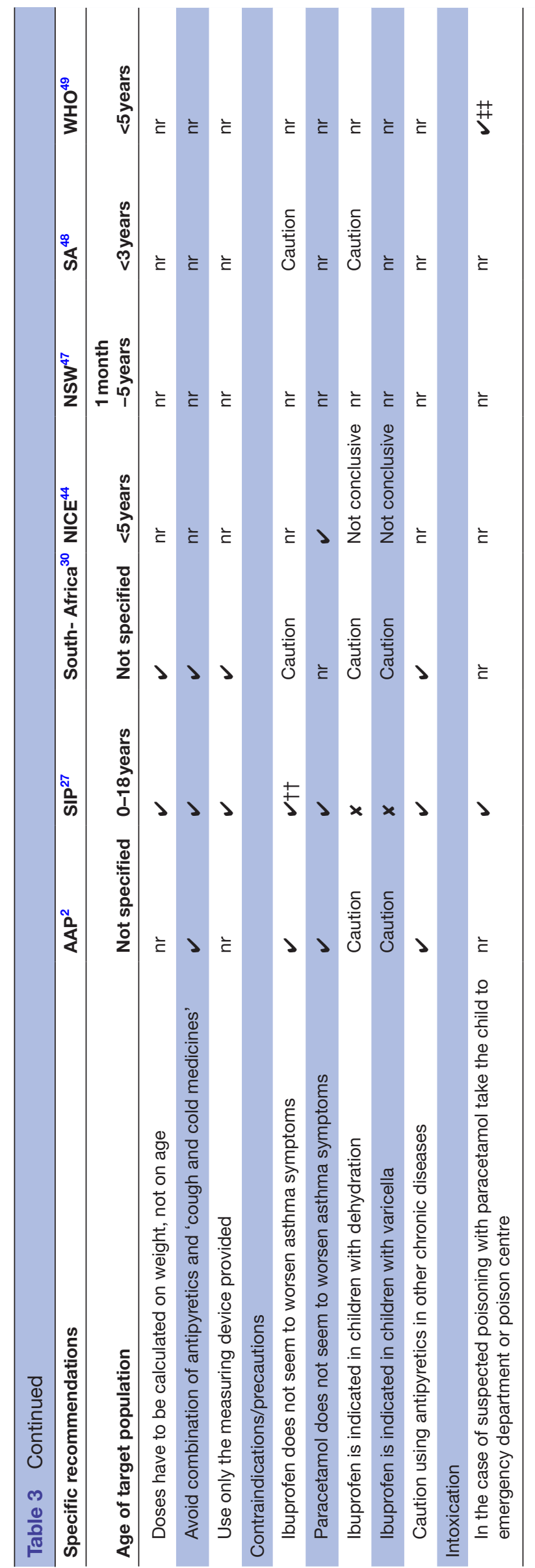

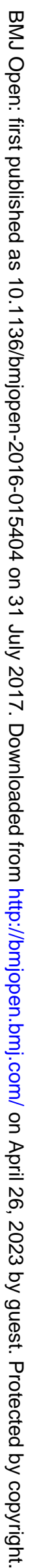


Interestingly, lower quality scores were observed in the domains of methodology, applicability and editorial independence, whereas all the guidelines had moderate to high scores in the purpose and objective and the clarity of the recommendations. The methodology analysis showed acceptable results only for the SIP, ${ }^{27}$ NICE, ${ }^{44}$ and WHO guidelines ${ }^{49}$ whereas the AAP, ${ }^{2}$ South African, ${ }^{30} \mathrm{NWS}^{47}$ and SA guidelines ${ }^{48}$ had insufficient scores because they were not based on a systematic review of the literature and they did not provide recommendations explicitly linked to evidence, or because they did not involve all the required members in the guideline development group.

Regarding the 'applicability' item, most guidelines did not describe the facilitators and barriers to their application and did not provide audit criteria. Some CPGs lacked a summary document, and educational tools. ${ }^{2}{ }^{30}$ This finding is in contrast with the need for clarity and user friendliness suggested by some authors ${ }^{19-22}$ and should be taken into consideration when developing new guidelines. Information on editorial independence was also neglected in most guidelines. ${ }^{30} 444788$ Indeed only AAP, SIP and WHO guidelines reported detailed information on potential conflicts of interest. ${ }^{2749}$ This is particularly important considering that conflicts of interest are the most common source of bias in guideline development. ${ }^{49}$ One strength of our study is that we used the AGREE II instrument for the first time to assess the methodological quality of guidelines related to fever in children.

The AGREE II instrument is a tool that assesses the methodological rigour and transparency with which a guideline is developed. ${ }^{23}$ A potential limitation of this method is that there is no threshold for distinguishing between high quality and low quality CPGs. Thus, the guideline quality would be left to the appraisers to identify and the scores of an AGREE II evaluation have to be interpreted with caution and confined to a particular situation. Furthermore, AGREE II does not consider the relative importance of the six domains of quality: rigour of development is considered of equal importance to the other five domains. ${ }^{24}$ This suggests that the domains of AGREE II should not be weighed equally. If the guideline has a low score on the domain of rigour of development, the corresponding recommendations have a high risk of bias, and the other domains are of little relevance in quality assessment. Another possible limitation of our study is that some guidelines may have been missed by our research.

From a comparison of the recommendations we observe that the guidelines agree on some crucial aspects. In particular, all guidelines agree on prescribing antipyretics only with the aim of relieving the child's discomfort caused by fever and not with the sole aim of reducing body temperature. There is also an agreement on the type of recommended antipyretics which are paracetamol or ibuprofen, according to a child's age, weight and characteristics. Several studies demonstrated the high tolerability of both these drugs with similar efficacy in reducing body temperature. ${ }^{79}$ However, few data are available regarding the impact of antipyretics in reducing the child's discomfort. ${ }^{79}$ The concept of 'discomfort' is not easy to define because it varies across different age groups, and may be influenced by the caregiver's perception. Therefore, future research needs to define child discomfort more precisely, and how to measure it using standardised clinical scores.

On the other hand, some messages diverge, either about the use of physical methods or the pharmacological approach. The common physical method to unwrap/ uncover children with fever is contraindicated by the SIP, South African and NICE CPGs, ${ }^{27} 3044$ but it is considered in the NWS and WHO CPGs. ${ }^{47} 49$ Furthermore, most guidelines discourage the alternate/combined use of two antipyretics except the NICE and SA CPGs that allow the alternate use of the two drugs, only when distress persists after the administration of one antipyretic. ${ }^{44}$ Alternate use can be associated with better control of body temperature, but no study demonstrated superiority in terms of distress control compared with monotherapy. However, it is certain that alternating two drugs increases the risk of overdose and toxicity. ${ }^{9}$ A greater consensus from the international scientific community would be desirable on a topic of such wide resonance.

In conclusion, most guidelines issued at national or international levels were of good quality and could be adopted in clinical practice. Nevertheless, the quality of the CPGs could be improved, in particular the methodological, applicability and editorial independence domains. Our study reinforces the messages of concordant recommendations and shines a light on discordant suggestions, and could stimulate the issues of international guidelines in order to unify medical behaviour.

Contributors EC conceptualised and designed the study, used AGREE II instrument to evaluate the retrieved guidelines, drafted the initial manuscript, and approved the final manuscript as submitted. BB carried out the initial analysis, used AGREE II instrument to evaluate the retrieved guidelines, drafted the initial manuscript, and approved the final manuscript as submitted. LG critically reviewed the manuscript, and approved the final manuscript as submitted. MdM critically reviewed the manuscript, and approved the final manuscript as submitted. All authors approved the final manuscript as submitted and agree to be accountable for all aspects of the work.

\section{Competing interests None declared.}

Patient consent The study does not includes patient data.

Provenance and peer review Not commissioned; externally peer reviewed. Data sharing statement No additional data available.

Open Access This is an Open Access article distributed in accordance with the Creative Commons Attribution Non Commercial (CC BY-NC 4.0) license, which permits others to distribute, remix, adapt, build upon this work non-commercially, and license their derivative works on different terms, provided the original work is properly cited and the use is non-commercial. See: http://creativecommons.org/ licenses/by-nc/4.0/

(c) Article author(s) (or their employer(s) unless otherwise stated in the text of the article) 2017. All rights reserved. No commercial use is permitted unless otherwise expressly granted.

\section{REFERENCES}

1. Crocetti M, Moghbeli N, Serwint J. Fever phobia revisited: have parental misconceptions about fever changed in 20 years? Pediatrics 2001;107:1241-6. 
2. Sullivan JE, Farrar HC. Section on clinical pharmacology and therapeutics, Committee on Drugs, fever and antipyretic use in children. Pediatrics 2011;127:580-7.

3. Bertille N, Fournier-Charrière E, Pons G, et al. Managing fever in children: a national survey of parents' knowledge and practices in France. PLoS One 2013;8:e83469.

4. Karwowska A, Nijssen-Jordan C, Johnson D, et al. Parental and health care provider understanding of childhood fever: a Canadian perspective. CJEM 2002;4:394-400.

5. Betz MG, Grunfeld AF. 'Fever phobia' in the emergency department: a survey of children's caregivers. Eur J Emerg Med 2006;13:129-33.

6. Lubrano R, Paoli S, Bonci M, et al. Acetaminophen administration in pediatric age: an observational prospective cross-sectional study. Ital J Pediatr 2016;42:20.

7. Kramer MS, Naimark LE, Roberts-Bräuer R, et al. Risks and benefits of paracetamol antipyresis in young children with fever of presumed viral origin. Lancet 1991;337:591-4.

8. Richardson M, Lakhanpaul M. Guideline Development Group and the Technical Team. Assessment and initial management of feverish illness in children younger than 5 years: summary of NICE guidance. BMJ 2007:334:1163-4.

9. Hay AD, Costelloe C, Redmond NM, et al. Paracetamol plus ibuprofen for the treatment of fever in children (PITCH): randomised controlled trial. BMJ 2008;337:a1302.

10. Bilenko N, Tessler H, Okbe R, et al. Determinants of antipyretic misuse in children up to 5 years of age: a cross-sectional study. Clin Ther 2006;28:783-93.

11. Matziou V, Brokalaki H, Kyritsi $\mathrm{H}$, et al. What Greek mothers know about evaluation and treatment of fever in children: an interview study. Int J Nurs Stud 2008;45:829-36.

12. Boivin JM, Weber F, Fay R, et al. [Management of paediatric fever: is parents' skill appropriate?]. Arch Pediatr 2007;14:322-9.

13. Impicciatore $P$, Nannini $S$, Pandolfini $C$, et al. Mother's knowledge of attitudes toward, and management of fever in preschool children in Italy. Prev Med 1998;27:268-73.

14. Langer T, Pfeifer M, Soenmez A, et al. Fearful or functional-a cross-sectional survey of the concepts of childhood fever among German and Turkish mothers in Germany. BMC Pediatr 2011;11:41.

15. Taveras EM, Durousseau S, Flores G. Parents' beliefs and practices regarding childhood fever: a study of a multiethnic and socioeconomically diverse sample of parents. Pediatr Emerg Care 2004;20:579-87.

16. Lava SA, Simonetti GD, Ramelli GP, et al. Symptomatic management of fever by Swiss board-certified pediatricians: results from a cross-sectional, web-based survey. Clin Ther 2012;34:250-6.

17. Chiappini E, Parretti A, Becherucci P, et al. Parental and medical knowledge and management of fever in Italian pre-school children. BMC Pediatr 2012;12:97.

18. Martinot A, Cohen R. [Attributes of elaboration and dissemination strategies that influence the implementation of clinical practice guidelines]. Arch Pediatr 2008;15:656-8.

19. May A, Bauchner H. Fever phobia: the pediatrician's contribution. Pediatrics 1992;90:851-4.

20. Cabana MD, Rand CS, Powe NR, et al. Why don't physicians follow clinical practice guidelines? A framework for improvement. JAMA 1999;282:1458-65.

21. Cavazos JM, Naik AD, Woofter A, et al. Barriers to physician adherence to nonsteroidal anti-inflammatory drug guidelines: a qualitative study. Aliment Pharmacol Ther 2008;28:789-98.

22. Dans AL, Dans LF. Appraising a tool for guideline appraisal (the AGREE II instrument). J Clin Epidemiol 2010;63:1281-2.

23. Ye ZK, Liu Y, Cui XL, et al. Critical appraisal of the Quality of Clinical Practice guidelines for stress ulcer prophylaxis. PLoS One 2016;11:e0155020.
24. Holmer HK, Ogden LA, Burda BU, et al. Quality of clinical practice guidelines for glycemic control in type 2 diabetes mellitus. PLoS One 2013;8:e58625

25. Wong T, Stang AS, Ganshorn H, et al. Cochrane in context: combined and alternating paracetamol and ibuprofen therapy for febrile children. Evid Based Child Health 2014;9:730-2.

26. Chiappini E, Principi N, Longhi R, et al. Management of fever in children: summary of the Italian Pediatric Society guidelines. Clin Ther 2009;31:1826-43.

27. Hoover L. AAP reports on the use of antipyretics for fever in children. Am Fam Physician 2012;85:518-9.

28. van den Anker JN. Optimising the management of fever and pain in children. Int J Clin Pract Suppl 2013;67:26-32.

29. Green R, Jeena P, Kotze S, et al. Management of acute fever in children: guideline for community healthcare providers and pharmacists. S Afr Med J 2013;103:948-54.

30. McIntyre J. Management of fever in children. Arch Dis Child 2011:96:1173-4.

31. Paul SP, Mayhew J, Mee A. Safe management and prescribing for fever in children. Nurse Prescribing 2011;9:539-44.

32. Pereira GL, Tavares NU, Mengue SS, et al. Therapeutic procedures and use of alternating antipyretic drugs for fever management in children. J Pediatr 2013;89:25-32.

33. De Ronne N. [Management of fever in children younger then 3 years]. J Pharm Belg 2010;3:53-7.

34. Reshadat S, Shakibaei D, Rezaei M, et al. Fever management in parents who have children aged 0-5 years. Scientific Journal of Hamadan University of Medical Sciences \& Health Services 2012;19:28-33.

35. Paul SP, Mee A, Mayhew J. The management of fever in children. Independent Nurse 2012;2012.

36. Sallam SA, El-Mazary AA, Osman AM, et al. Integrated management of childhood illness (IMCl) approach in management of children with high grade fever $\geq 39^{\circ}$. Int J Health Sci 2016;10:239-48.

37. Pirker A, Pirker M. [Management of fever in children]. Ther Umsch 2015;72:15-17.

38. Tantracheewathom T. Fever of unknown origin in children: approach and management: Vajira Medical Journal, 2010.

39. Walls T. Evaluation and management of fever in children. $N Z$ Med J 2010;123:15-17.

40. Long SS. Diagnosis and management of undifferentiated fever in children. J Infect 2016:72(Suppl: 68-76):S68-S76.

41. Long D, Flatley C, Williams T, et al. Occurrence, management and outcome of fever in critically ill children. Australian Critical Care 2016;29:123.

42. Medical City King Saud University (KSUMC. Evidence-based clinical practice guideline for management of fever of uncertain source in infants 60 days of age or less. 1st Edition, 2013.

43. National Institute for Health and Care Excellence. Feverish illness in children: assessment and initial management in children younger than five years, 2013.

44. M29 NHG Clinical Practice Guideline Feverish illness in Children. BohnStafleu van Loghum. The Netherlands: Part of Springer Media, 2011.

45. Cincinnati Children's Hospital Medical Center. Evidence based clinical practice guideline for fever of uncertain source in children 2 to 36 months of age, 2010.

46. New South Wales (NSW) Kids and Families. Children and Infants with fever - Acutemanagement, 2010.

47. SA Child Health Clinical Network. Fever without a focus in infants and children excluding the newborn, 2013.

48. World Health Organization. Pocket book of Hospital Care for Children: guidelines for the management of Common Childhood Illnesses, 2013.

49. Detsky AS. Sources of bias for authors of clinical practice guidelines. CMAJ 2006;175:1033-5. 